\title{
Impedance Eduction in Ducts with Higher-Order Modes and Flow
}

\author{
W. R. Watson* and M. G. Jones ${ }^{\dagger}$ \\ NASA Langley Research Center, Hampton, Virginia 23681-2199, USA
}

\begin{abstract}
An impedance eduction technique, previously validated for ducts with plane waves at the source and duct termination planes, has been extended to support higher-order modes at these locations. Inputs for this method are the acoustic pressures along the source and duct termination planes, and along a microphone array located in a wall either adjacent or opposite to the test liner. A second impedance eduction technique is then presented that eliminates the need for the microphone array. The integrity of both methods is tested using three sound sources, six Mach numbers, and six selected frequencies. Results are presented for both a hardwall and a test liner (with known impedance) consisting of a perforated plate bonded to a honeycomb core. The primary conclusion of the study is that the second method performs well in the presence of higher-order modes and flow. However, the first method performs poorly when most of the microphones are located near acoustic pressure nulls. The negative effects of the acoustic pressure nulls can be mitigated by a judicious choice of the mode structure in the sound source. The paper closes by using the first impedance eduction method to design a rectangular array of 32 microphones for accurate impedance eduction in the NASA LaRC Curved Duct Test Rig in the presence of expected measurement uncertainties, higher order modes, and mean flow.
\end{abstract}

\section{Introduction}

$I^{\mathrm{r}}$ NCREASINGLY stringent noise constraints in the United States and abroad have resulted in continued emphasis on the development of improved acoustic liner technologies to reduce the overall levels of fan noise radiated to communities surrounding airports. Consequently, various numerical methods have been developed by government, industry, and academia to support locally-reacting liner impedance evaluation. This procedure is commonly referred to as impedance eduction although some researchers abroad refer to it as "impedance determination using an inverse method." The numerical methods ${ }^{1-14}$ include codes based on modal and finite element analyses. Of these, finite element codes developed at the NASA Langley Research Center (LaRC) have been the most thoroughly tested in the open literature ${ }^{4,5,11,13}$ and have been demonstrated to provide quality results in ducts where a single plane wave mode can be isolated between a pair of opposite hard walls.

A number of experimental test rigs have also been used by the U.S. aircraft industry to evaluate locally-reacting liners using various levels of detail. Of particular note are the Spirit Aerosystems (formerly a Boeing facility), ${ }^{6}$ the United Technology Research Center flow impedance tubes, ${ }^{15}$ and the Goodrich Aerostructures insertion loss facility. ${ }^{6}$ Further, over the last three decades LaRC has developed a number of test rigs for the evaluation of locally-reacting acoustic liners in the presence of grazing flow. The most notable of these are the Grazing Incidence Tube (GIT) ${ }^{16}$ and the Curved Duct Test Rig (CDTR). ${ }^{17}$

Impedances are educed for test liners in the GIT using the following four-step procedure: ${ }^{4}$

1. Measure the plane wave acoustic pressures at a microphone array distributed along the lower wall in the hardwall section upstream of the test liner.

2. Measure a near anechoic duct termination impedance in the hardwall section downstream of the test liner.

3. Measure the acoustic pressures at a microphone array distributed along a hardwall opposite the test liner.

\footnotetext{
*Senior Research Scientist, Research and Technology Directorate, Computational AeroSciences Branch, Liner Physics Group, Associate Fellow, AIAA.

${ }^{\dagger}$ Senior Research Scientist, Research and Technology Directorate, Structural Acoustics Branch, Head Liner Physics Group, Associate Fellow AIAA.
} 
4. Use a duct propagation model based upon a finite-element methodology to educe the liner impedance by iterating on an assumed value of test liner impedance (via a gradient based optimizer) until the predicted and measured wall pressures are matched (within some tolerance) along the microphone array.

More recently, a new test rig has been built to replace the GIT. This rig, labeled the Grazing Flow Impedance Tube (GFIT), has a $63.5 \mathrm{~mm}$ by $51 \mathrm{~mm}$ cross-section and supports single-tone sources of up to $145 \mathrm{~dB}$ over a frequency range of 0.4 to $3.0 \mathrm{kHz}$, with mean flow Mach numbers up to 0.5 .

It was clear from the onset of the current effort that the impedance eduction methodology described above for the GIT and currently modified for the GFIT, is not suited for application to the CDTR. This occurs for two reasons. First, the dimensions of the CDTR are closer to those of a full-scale engine; therefore, the source and exit planes must support higher-order modes (the GIT is generally operated at frequencies where no higher-order modes propagate in hardwall sections). Second, the wall opposite the test liner in the CDTR was designed to contain another liner and populating it with a microphone array (as in the GIT and GFIT) is not convenient.

This paper has three primary objectives. First, the impedance eduction methodology used in the $\mathrm{GIT}^{4,5,11,13}$ (and now the GFIT) is extended to support higher-order mode inputs in the hardwall sections upstream and downstream of the test liner. Second, an acceptable microphone array is designed for the CDTR that can be used to educe liner impedance in the presence of higher-order modes, mean flow, and measurement uncertainties. The microphone array is imbedded in the hardwall adjacent to the test liner. Third, an impedance eduction methodology is presented for ducts in which the need for a microphone array imbedded in a wall opposite or adjacent to the test liner is eliminated.

\section{Description of Duct Configurations}

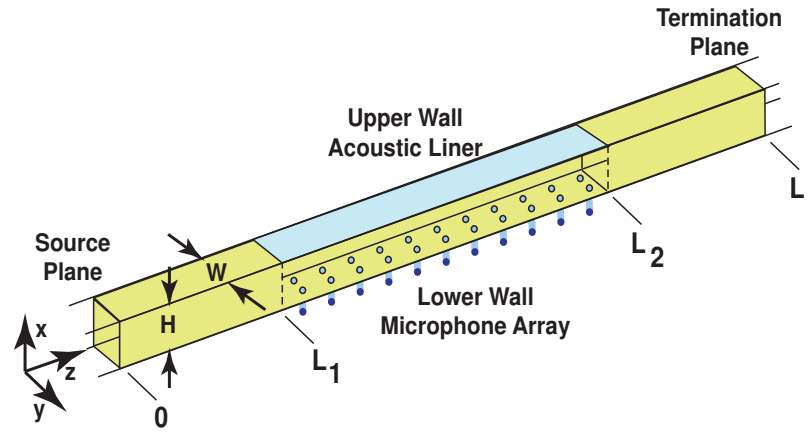

(a) Configuration 1: microphone array located on opposite wall

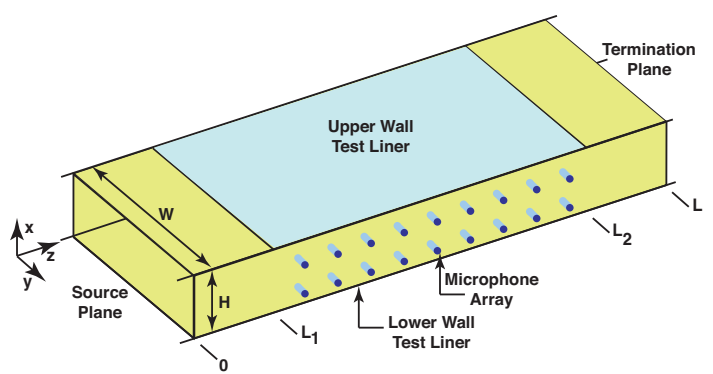

(b) Configuration 2: microphone array located on adjacent wall

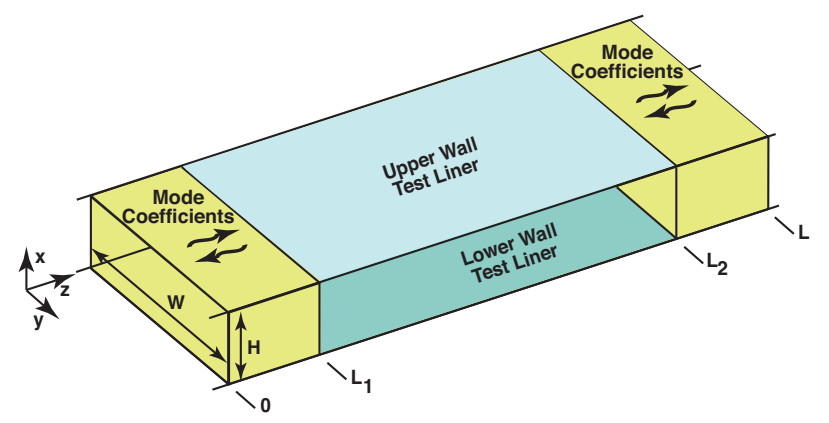

(c) Configuration 3: microphone array eliminated

Figure 1. Schematic of 3D duct configurations.

Figure 1 shows the three duct configurations for which impedance eduction is desired. Each configuration contains hardwall sections upstream and downstream of the test liner/liners and the left and right sidewalls are rigid. The configuration depicted in Fig. 1a (for which the microphone array in the liner test section is flush-mounted into the hardwall opposite the test liner) is consistent with the current GFIT setup. This configuration has been thoroughly tested for a plane acoustic wave at the source plane and a near anechoic termination impedance. An array of flush-mounted 
microphones (not shown in Fig. 1) is used to measure the source plane pressure and duct termination impedance in the hardwall sections upstream and downstream of the test liners. In a recent study, ${ }^{13}$ it was shown that a termination pressure boundary condition gives more stable and consistent results than a boundary condition based on the exit impedance. Thus, the current formulation incorporates an acoustic pressure condition as the termination boundary condition. Both the source and duct termination boundary conditions are then modified to account for higher-order mode effects. The configuration illustrated in Fig. 1b (in which a second liner is located on the wall opposite the test liner and the microphone array is flush-mounted into a rigid sidewall adjacent to the test liner/liners) is consistent with the current setup of the CDTR. This configuration has been evaluated for a plane wave source and planar exit impedance, but has not been tested in the presence of higher-order modes. This paper, therefore, tests the integrity of this configuration for impedance eduction in the presence of higher-order modes and flow. We also demonstrate how to design a microphone array for this configuration that is well suited for impedance eduction. The third configuration (in which a microphone array imbedded in an opposite or adjacent wall to the test liner/liners is eliminated) is depicted in Fig. 1c. An impedance eduction theory for the third configuration is not available in the duct acoustics literature and is presented (and tested) in this paper for the first time.

In each of the three duct configurations a right-hand Cartesian coordinate system is used in which the axial, vertical, and horizontal directions are denoted by $z, x$, and $y$, respectively. Each duct has length $L$, uniform height $H$ and uniform width $W$. However, the cross-sectional dimensions of the GIT and GFIT are considerably smaller than those of the CDTR. Although the height and width of each configuration is depicted as constant, provisions will be made later to allow for a nonuniform cross-sectional area distribution between the uniform hardwall sections upstream and downstream of the test liner/liners. Such a nonuniform area distribution exists, for example, when the CDTR is operated in a curved configuration.

As usual, all transients in the ducts are assumed to have been dissipated away or to have propagated out of the solution domain, so that acoustic disturbances are assumed to be at steady-state with a harmonic time dependence of the form $e^{i 2 \pi f t}$, where $f$ is the source frequency in Hertz, $t$ denotes the time in seconds, and $i$ is the unit imaginary number. The source and duct termination planes are located at $z=0$ and $z=L$, respectively, and the origin of the coordinate system is the lower left corner of the source plane as illustrated in Fig. 1. Note that the left and right sidewall of each duct is rigid and the upper wall always contains a test liner. Throughout this paper it will be assumed that the lower wall in configuration 1 is rigid, whereas configurations 2 and 3 may contain a second liner on the lower wall. In each configuration, the liner/liners lie between the axial locations, $L_{1} \leq z \leq L_{2}$, and outside of this region the upper and lower walls are rigid. All liners are assumed to be locally reacting (i.e., acoustic waves propagate through them normal to the surface). The impedance of lower and upper wall liners are denoted by $\zeta_{1}(z)$ and $\zeta_{2}(z)$, respectively, so that the impedance of each liner may vary with axial location. Again it is emphasized that the lower wall of configuration 1a is rigid (i.e., $1 / \zeta_{1}(z)=0.0$ ). Further, in this paper all impedances are assumed normalized with respect to the characteristic impedance of the air in the duct and all variables are in the International System of Units (unless otherwise specified).

In each duct, there is a subsonic mean flow from left to right along the axis of the duct with speed, $U_{0}$. The mean flow is not shown in Fig. 1 but is assumed present. The boundary layer generated by the flow is assumed thin, so that the uniform flow assumption is acceptable. Therefore, the mean flow field is assumed to have a constant mean static density of $\rho_{0}$, a constant mean static pressure of $P_{0}$, and a constant mean static temperature of $T_{0}$. Further, each duct configuration is a model of an aft-fan duct (i.e., sound and flow are in the same direction). Alternatively, inlet duct modeling (i.e., flow and sound directed in opposite directions) is achieved by reversing the sign on the steady flow velocity, $U_{0}$. The problem at hand is to educe the unknown acoustic impedance of the test liner/liners for each of the three duct configurations, in the presence of the uniformly flowing fluid and higher-order modes.

\section{Governing Equations and Boundary Conditions}

The acoustic pressure field, $p$, propagating in the ducts depicted in Fig. 1 satisfies the convected wave equation

$$
\left(1-M_{0}^{2}\right) \frac{\partial^{2} p}{\partial z^{2}}+\frac{\partial^{2} p}{\partial x^{2}}+\frac{\partial^{2} p}{\partial y^{2}}-2 i k M_{0} \frac{\partial p}{\partial z}+k^{2} p=0
$$

where $p$ is the acoustic pressure field, $k=(2 \pi f) / c_{0}$, is the freespace wavenumber, $M_{0}=U_{0} / c_{0}$, is the mean flow Mach number, and $c_{0}$ is the speed of sound in the duct. A detailed derivation of Eq. (1) along with the simplifying assumptions are given in Ref. 18. The solution to Eq. (1) that satisfies the rigid sidewall boundary conditions is of the form 


$$
p(z, x, y)=\sum_{m=0}^{\bar{M}-1} p_{m}(z, x) \cos \left(\frac{m \pi y}{W}\right)
$$

where the upper limit of summation, $\bar{M}-1$, is chosen to include each horizontal mode that propagates without decay in the hardwall sections of duct. In addition the horizontal mode order, $m$, is assumed a known integer, so that each $p_{m}$ is a quasi-3D solution. Because the solution in the horizontal dimension is separable, each quasi-3D solution, $p_{m}$, in Eq. (2) can be shown to satisfy a modified convected wave equation of the form

$$
\left(1-M_{0}^{2}\right) \frac{\partial^{2} p_{m}}{\partial z^{2}}+\frac{\partial^{2} p_{m}}{\partial x^{2}}-2 i k M_{0} \frac{\partial p_{m}}{\partial z}+\left[k^{2}-\left(\frac{m \pi}{W}\right)^{2}\right] p_{m}=0
$$

The wall impedance boundary condition for the locally-reacting liner has been discussed in detail by Myers. ${ }^{19}$ For the upper wall, this condition is

$$
-\frac{\partial p_{m}}{\partial x}=i k\left(\frac{p_{m}}{\zeta_{2}}\right)+2 M_{0} \frac{\partial}{\partial z}\left(\frac{p_{m}}{\zeta_{2}}\right)+\frac{M_{0}^{2}}{i k} \frac{\partial^{2}}{\partial z^{2}}\left(\frac{p_{m}}{\zeta_{2}}\right)
$$

and the wall impedance boundary condition of the lower wall has a similar form

$$
\frac{\partial p_{m}}{\partial x}=i k\left(\frac{p_{m}}{\zeta_{1}}\right)+2 M_{0} \frac{\partial}{\partial z}\left(\frac{p_{m}}{\zeta_{1}}\right)+\frac{M_{0}^{2}}{i k} \frac{\partial^{2}}{\partial z^{2}}\left(\frac{p_{m}}{\zeta_{1}}\right)
$$

Equations (4) and (5) are also used along the hardwall portion of the upper and lower wall, but the reciprocal of the impedance functions are set to zero [i.e., $1 / \zeta_{1}(z)=1 / \zeta_{2}(z)=0.0$ ] along this portion of the wall.

An array of microphones flush-mounted in the hardwall section upstream of the test liners (not shown in the enclosed figures) is used to measure the acoustic pressure profile, $p_{s}(x)$, at the source plane for each horizontal mode, $m$. This measured acoustic pressure profile is used as the source plane boundary condition

$$
p_{m}(0, x)=p_{s}(x)
$$

Similarly, the microphone array in the hardwall section downstream of the test liners (also not shown in the enclosed figures) is used to determine the exit plane acoustic pressure boundary condition, $p_{e}(x)$, for each horizontal mode

$$
p_{m}(L, x)=p_{e}(x)
$$

Note that the acoustic pressure profile measurements must be obtained with the test liner/liners installed and must contain the effects of higher-order modes in the vertical direction (i.e., the $x$ direction). Equations (3)-(7) do not have exact closed-form solutions for a general set of input parameters. Consequently, they are solved numerically using a finite element method. A few of the most important details concerning the finite element method are given in the following section.

\section{Finite Element Solution for the Acoustic Pressure Field}

In the ducts depicted in Fig. 1, a grid consisting of $N$ and $M$ evenly spaced grid lines is used in the $z$ and $x$ directions of the duct, respectively. The $N$ grid lines in the $z$ direction are assumed to be located at axial locations $z=z_{1}, z=z_{2}, \ldots z=z_{N}$ (where $z_{1}=0$, and $z_{N}=L$ ). Similarly, the $M$ grid lines in the $x$ direction are assumed to be located at $x=x_{1}, x=x_{2}, \ldots x=x_{M}$ (where $x_{1}=0$, and $x_{M}=H$ ). Thus, there are $N-1$ columns and $M-1$ rows of elements in the duct as shown in the upper part of Fig. 2. It is convenient therefore to use a double subscript notation, $[I, J]$, to locate the element in the $I$ th column and $J$ th row of the grid, where $I=1,2,3 \ldots N-1$ and $J=1,2,3 \ldots M-1$. A typical rectangular element, $[I, J]$, with width $a=z_{I+1}-z_{I}$ and height $b=x_{J+1}-x_{J}$ consists of four local node numbers, labeled 1,2,3, and 4, respectively, as shown in the bottom part of Fig. 2. The objective is to obtain the unknown acoustic pressure and its derivatives at the nodes of each of the $(M-1)(N-1)$ rectangular elements. This is achieved by using a Galerkin finite-element method to minimize the field residual error, which is defined as

$$
R_{F}(z, x)=\left(1-M_{0}^{2}\right) \frac{\partial^{2} p_{m}}{\partial z^{2}}+\frac{\partial^{2} p_{m}}{\partial x^{2}}-2 i k M_{0} \frac{\partial p_{m}}{\partial z}+\left[k^{2}-\left(\frac{m \pi}{W}\right)^{2}\right] p_{m}
$$



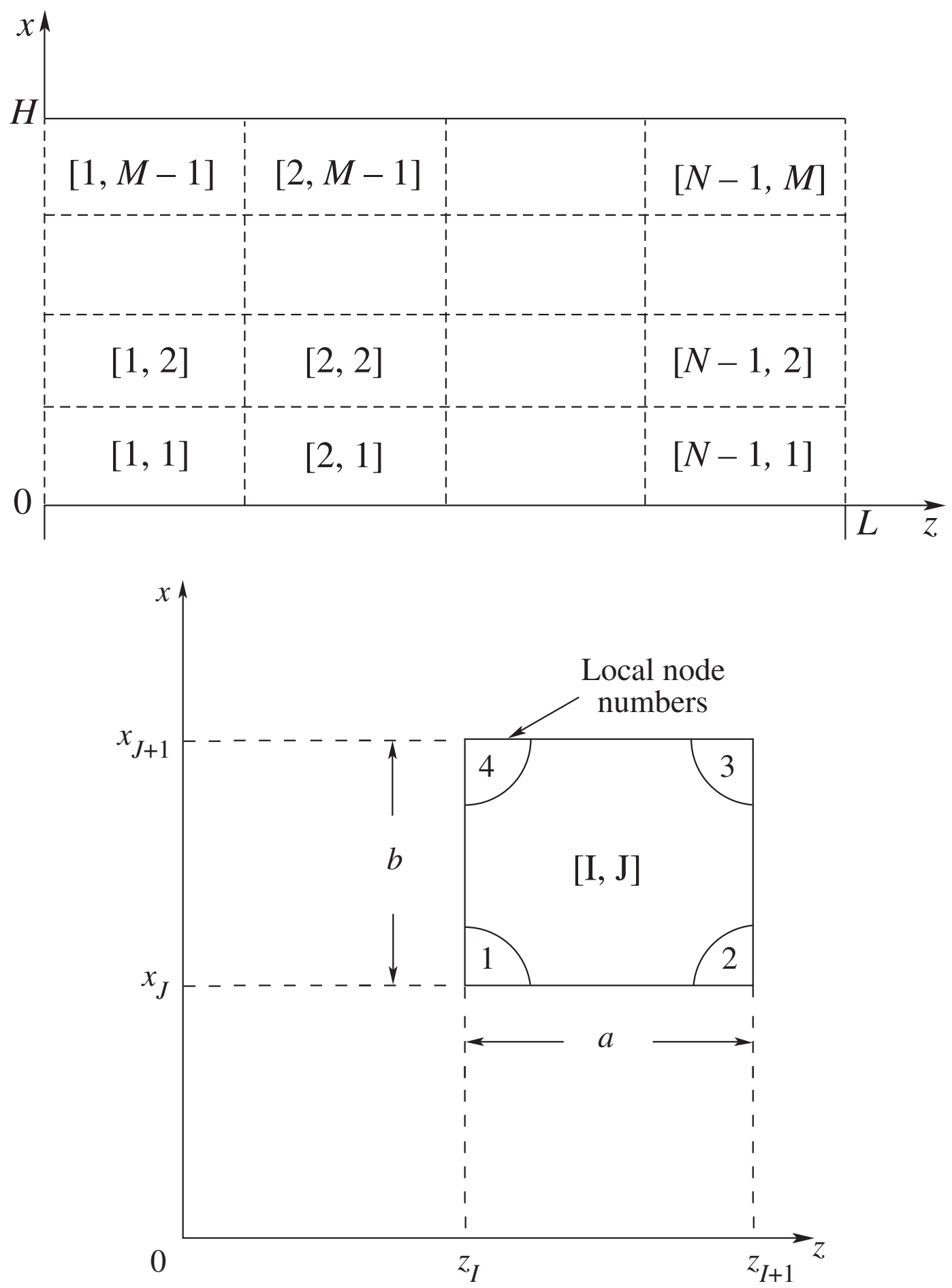

Figure 2. Finite element discretization and node numbering system. 
Within each rectangular element, $[I, J]$, the acoustic pressure, $p_{m}$, is approximated as a cubic polynomial

$$
p_{m}(z, x)=\sum_{q=1}^{q=16} N_{q}(z, x) p_{q}
$$

Expressions for the cubic polynomial basis functions, $N_{q}(z, x)$, are somewhat lengthy and can be found in standard finite element textbooks such as Ref. 20. Here, we used a $C^{1}$ continuous element ${ }^{20}$ so that the nodal coefficients, $p_{q}$, are

$$
\begin{array}{llll}
p_{1}=p_{m}\left(z_{I}, x_{J}\right) & p_{5}=p_{m}\left(z_{I+1}, x_{J}\right) & p_{9}=p_{m}\left(z_{I+1}, x_{J+1}\right) & p_{13}=p_{m}\left(z_{I}, x_{J+1}\right) \\
p_{2}=\frac{\partial p_{m}\left(z_{I}, x_{J}\right)}{\partial z} & p_{6}=\frac{\partial p_{m}\left(z_{I+1}, x_{J}\right)}{\partial z} & p_{10}=\frac{\partial p_{m}\left(z_{I+1}, x_{J+1}\right)}{\partial z} & p_{14}=\frac{\partial p_{m}\left(z_{I}, x_{J+1}\right)}{\partial z} \\
p_{3}=\frac{\partial p_{m}\left(z_{I}, x_{J}\right)}{\partial x} & p_{7}=\frac{\partial p_{m}\left(z_{I+1}, x_{J}\right)}{\partial x} & p_{11}=\frac{\partial p_{m}\left(z_{I+1}, x_{J+1}\right)}{\partial x} & p_{15}=\frac{\partial p_{m}\left(z_{I}, x_{J+1}\right)}{\partial x} \\
p_{4}=\frac{\partial^{2} p_{m}\left(z_{I}, x_{J}\right)}{\partial z \partial x} & p_{8}=\frac{\partial^{2} p_{m}\left(z_{I+1}, x_{J}\right)}{\partial z \partial x} & p_{12}=\frac{\partial^{2} p_{m}\left(z_{I+1}, x_{J+1}\right)}{\partial z \partial x} & p_{16}=\frac{\partial_{m}^{2}\left(z_{I}, x_{J+1}\right)}{\partial z \partial x}
\end{array}
$$

The unknown wall impedance functions $\zeta_{2}(z)$ and $\zeta_{1}(z)$ are expanded in series similar to Eq. (9). However, the coefficients in the expansion are the unknown nodal values of the wall impedance functions [i.e., $\zeta_{1}\left(z_{I}\right)$, and $\zeta_{2}\left(z_{I}\right)$ ] and these coefficients will need to be determined.

The field error in the duct is now minimized by requiring that it be orthogonal to each basis function, $N_{q}(z, x)$. Because each element is assumed evenly spaced, contributions to the minimization of the field error function for each element can be computed from that of a single finite element such as element $[1,1]$. This contribution is of the form

$$
\int_{[1,1]} R_{F} N_{q} d z d x
$$

Each second-derivative term in the integrand of equation (11) is integrated by parts to obtain

$$
\begin{gathered}
\int_{[1,1]} R_{F} N_{q} d z d x=\int_{0}^{b} \int_{0}^{a} R_{F} N_{q} d z d x=R_{1}+R_{2}+R_{z}+R_{x} \\
R_{1}=\int_{0}^{b} \int_{0}^{a}\left[-\left(1-M_{0}^{2}\right) \frac{\partial p_{m}}{\partial z} \frac{\partial N_{q}}{\partial z}-\frac{\partial p_{m}}{\partial x} \frac{\partial N_{q}}{\partial x}-2 i k M_{0} \frac{\partial p_{m}}{\partial z} N_{q}\right] d z d x \\
R_{2}=\int_{0}^{b} \int_{0}^{a}\left[k^{2}-\left(\frac{m \pi}{W}\right)^{2}\right] p_{m} N_{q} d z d x \\
R_{z}=\int_{0}^{b}\left(1-M_{0}^{2}\right) \frac{\partial p_{m}(a, x)}{\partial z} N_{q}(a, x) d x-\int_{0}^{b}\left(1-M_{0}^{2}\right) \frac{\partial p_{m}(0, x)}{\partial z} N_{q}(0, x) d x \\
R_{x}=\int_{0}^{a}\left[\frac{\partial p_{m}(z, b)}{\partial x} N_{q}(z, b)\right] d z-\int_{0}^{a}\left[\frac{\partial p_{m}(z, 0)}{\partial x} N_{q}(z, 0)\right] d z
\end{gathered}
$$

where $a$ and $b$ are the width and height, respectively, of the finite element as shown in Fig. 2. The wall impedance boundary conditions are implemented into the boundary integrals in Eq. (16)

$$
\begin{gathered}
\int_{0}^{a}\left[\frac{\partial p_{m}}{\partial x} N_{q}\right] d z=-\int_{0}^{a}\left[i k\left(\frac{p_{m}}{\zeta_{2}}\right)+2 M_{0} \frac{\partial}{\partial z}\left(\frac{p_{m}}{\zeta_{2}}\right)+\frac{M_{0}^{2}}{i k} \frac{\partial^{2}}{\partial z^{2}}\left(\frac{p_{m}}{\zeta_{2}}\right)\right] N_{q} d z, x=a \\
\int_{0}^{a}\left[\frac{\partial p_{m}}{\partial x} N_{q}\right] d z=\int_{0}^{a}\left[i k\left(\frac{p_{m}}{\zeta_{1}}\right)+2 M_{0} \frac{\partial}{\partial z}\left(\frac{p_{m}}{\zeta_{1}}\right)+\frac{M_{0}^{2}}{i k} \frac{\partial^{2}}{\partial z^{2}}\left(\frac{p_{m}}{\zeta_{1}}\right)\right] N_{q} d z, x=0
\end{gathered}
$$

The contribution to the minimization of the field error and wall impedance boundary conditions for a typical element, $[I, J]$, is expressed in matrix form

$$
\int_{[I, J]} R_{F} N_{q} d z d x=\left[A^{[I, J]}\right]\left\{P^{[I, J]}\right\}
$$

where $\left[A^{[I, J]}\right]$, is the $16 \times 16$ element stiffness matrix, and $\left\{P^{[I, J]}\right\}$ is a complex column vector of length 16 that contains the unknown acoustic pressure and its derivatives at the four nodes of element, $[I, J]$. The coefficients in the element stiffness matrix are computed in closed form. Assembly of the global matrix equations from the local element stiffness matrices is a basic procedure in the finite-element method. Appropriate shifting of rows and columns is all 
that is required to add the local element stiffness matrix $\left[A^{[I, J]}\right]$ directly into the global stiffness matrix, $[\bar{A}]$. This results in a matrix equation of the form

$$
[\bar{A}]\{P\}=\{0\}
$$

where $\{0\}$ is a null column vector of length $4 N M,[\bar{A}]$ is the $4 N M \times 4 N M$ block tridiagonal global stiffness matrix, and $\{P\}$ is a column vector of length $4 N M$ containing the unknown nodal coefficients. In Eq. (20) the vector $\{P\}$ is partitioned as follows

$$
\{P\}=\left\{\begin{array}{c}
\left\{P_{1}\right\} \\
\left\{P_{2}\right\} \\
\left\{P_{3}\right\} \\
\vdots \\
\left\{P_{N}\right\}
\end{array}\right\}
$$

were each $\left\{P_{I}\right\}$ is a column vector of length $4 M$ that contains the unknown nodal parameters at the axial gridline, $z=z_{I}$.

Equations (20) are a set of linear algebraic equations that minimize the residual error in the duct while satisfying the wall impedance boundary conditions in weak form. Solutions to Eqs. (20) may not, however, satisfy the source plane [Eq. (6)] and duct termination plane [Eq. (7)] boundary conditions. These two conditions are satisfied by constraining the nodal degrees of freedom, $p_{q}$, in the trial solution given in Eq. (9). The methodology for imposing these constraints on a linear system such as Eqs. (20) is found in standard finite element texts such as Ref. 20. Constraining the nodal degrees of freedom to satisfy the source and duct termination boundary condition leads to a modified system of linear equations of the form

$$
[A]\{P\}=\{F\}
$$

where $\{F\}$ is a column vector of length $4 N M$ that contains the effects of the sound source and duct termination acoustic pressure, and $[A]$ is the modified asymmetric stiffness matrix. Equation (22) constitutes the system of linear algebraic equations that are solved to educe the impedance for each of the three duct configurations. The impedance eduction methodology for duct configurations 1 and 2 are similar and are therefore described in the same section. However, the impedance eduction methodology for configuration 3 has not been presented in the open literature and is described in a separate section.

\section{Impedance Eduction for Configurations 1 and 2}

Given initial estimates for the impedance functions $\left[\zeta_{1}\left(z_{I}\right)\right.$, and $\left.\zeta_{2}\left(z_{I}\right)\right]$ at the finite element boundary nodes, solutions to Eq. (22) are used to obtain estimates for the acoustic pressure [i.e., $p_{E s t}\left(z_{I}\right)$ ] at axial locations, $z_{I}$, along the microphone array. Values of the unknown impedance functions and their derivatives at the finite element nodes are then iteratively updated until a set of impedance functions are found that reproduces the acoustic pressures, $p_{\text {Meas }}\left(z_{I}\right)$, measured at the microphone array to within some tolerance. Throughout this paper the superscript Meas and Est are used to denote a measured value and a value obtained from the solution to Eq. (22), respectively.

It is necessary, however, to automate the above iterative procedure so that $p_{E s t}\left(z_{I}\right)$ is closer to $p_{\text {Meas }}\left(z_{I}\right)$ for each new update of the impedance functions. We therefore implement an automated search procedure using an optimization algorithm. The objective function, $F_{\text {wall }}\left(\zeta_{1}, \zeta_{2}\right)$, for the optimization algorithm is defined as

$$
F_{\text {wall }}\left(\zeta_{1}, \zeta_{2}\right)=\left\|\left\{R_{\text {dif }}\right\}\right\|_{L 2}, \quad\left\{R_{\text {dif }}\right\}=\frac{1}{n \text { wall }}\left\{\begin{array}{c}
p_{\text {Meas }}\left(z_{1}\right)-p_{\text {Est }}\left(z_{1}\right) \\
p_{\text {Meas }}\left(z_{2}\right)-p_{E s t}\left(z_{2}\right) \\
p_{\text {Meas }}\left(z_{3}\right)-p_{E s t}\left(z_{3}\right) \\
\vdots \\
p_{\text {Meas }}\left(z_{\text {nwall }}\right)-p_{\text {Est }}\left(z_{\text {nwall }}\right)
\end{array}\right\}
$$

where $n$ wall is the number of axial locations, $z_{I}$, along the microphone array where measurements are taken, and $\left\|\left\{R_{\text {dif }}\right\}\right\|_{L 2}$ denotes the L2-norm of the residual pressure error vector

$$
\left\|\left\{R_{\text {dif }}\right\}\right\|_{L 2}=\left\{R_{\text {dif }}\right\}\left\{R_{\text {dif }}^{*}\right\}^{T}
$$

In Eq. (24) the superscripts $*$ and $T$ denote the complex conjugate and vector transpose, respectively. Note the following:

1. The objective function is real and nonnegative (i.e., $F_{\text {wall }}\left(\zeta_{1}, \zeta_{2}\right) \geq 0$ ) 
2. The global minimum of the objective function, $F_{\text {wall }}\left(\zeta_{1}, \zeta_{2}\right)=0$, occurs when $p_{E s t}\left(z_{I}\right)=p_{\text {Meas }}\left(z_{I}\right)$ for each $z_{I}$

Because the acoustic pressure at the microphone array is a unique function of the test liner impedance functions, it follows therefore that the impedance functions that minimize $F_{\text {wall }}\left(\zeta_{1}, \zeta_{2}\right)$ are the unknown impedance functions of the test liner. The optimization algorithm returns the impedance functions [that is, $\zeta_{1}(z)$ and $\zeta_{2}(z)$ ] that minimize this objective function.

\section{Impedance Eduction for Configuration 3}

In this section an impedance eduction technique is developed for application to the duct configuration depicted in Fig. 1c. Note that this configuration does not contain a microphone array in the walls opposite or adjacent to the test liners. Therefore an impedance eduction method different from that described in the previous section is required.

To begin, the solution to Eq. (22) gives unique values for the axial component of the acoustic pressure gradient, $\frac{\partial p_{m}}{\partial z}$, at the source and duct termination plane for each set of impedance functions. Further, the solution in the hardwall section upstream of the liner is expanded as a series of hardwall duct modes

$$
\begin{gathered}
p_{m}(z, x)=\sum_{n=0}^{\bar{N}-1}\left[A_{m, n}^{+} e^{i K_{m, n}^{+} z}+A_{m, n}^{-} e^{i K_{m, n}^{-} z}\right] \cos \left(\frac{n \pi x}{H}\right) \\
\frac{K_{m, n}^{ \pm}}{k}=\frac{M_{0} \pm \sqrt{1-\left(1-M_{0}^{2}\right)\left[(m \pi / k W)^{2}+(n \pi / k H)^{2}\right]}}{\left(1-M_{0}^{2}\right)}
\end{gathered}
$$

where the upper index of summation $[(\bar{N}-1)]$ is chosen so that each mode that propagates along the $z$ axis without axial decay is included in the series. Here, it is implicitly assumed that the source plane is located far enough upstream of the leading edge of the liner, that the axially decaying modes have decayed to sufficiently low levels to be neglected. Further, the right and left-moving mode coefficients (i.e., $A_{m, n}^{ \pm}$) are assumed known from acoustic measurements. Note that the left-moving mode coefficients, $A_{m, n}^{-}$, originate from the reflection of acoustic energy at the leading edge of the liner, while the right-moving mode coefficients, $A_{m, n}^{+}$, carry the predominant acoustic energy generated by the sound source. The solution in the downstream hardwall section is also expanded in a similar series of hardwall duct modes

$$
p_{m}(z, x)=\sum_{n=0}^{\bar{N}-1}\left[C_{m, n}^{+} e^{i K_{m, n}^{+} z}+C_{m, n}^{-} e^{i K_{m, n}^{-} z}\right] \cos \left(\frac{n \pi x}{H}\right)
$$

where the right and left moving mode coefficients (i.e., $C_{m, n}^{ \pm}$) are assumed known from acoustic measurements.

The impedance eduction method proposed for configuration 3 is based on the following two criteria

1. The boundary value problem defined by Eqs. (3)-(7) is well-posed. That is, the acoustic pressure and its gradients are uniquely determined by the test liner impedance functions, the source plane and exit plane acoustic pressure

2. The finite-element solution for the axial pressure gradients at the source and duct termination planes must match those obtained from the acoustic measurements

More specifically, the above two criteria require that the following equations be satisfied

$$
\begin{gathered}
\left\{\frac{\partial P 1}{\partial z}\right\}_{\text {Meas }}-\left\{\frac{\partial P 1}{\partial z}\right\}_{E s t}=\{0\} \\
\left\{\frac{\partial P N}{\partial z}\right\}_{\text {Meas }}-\left\{\frac{\partial P N}{\partial z}\right\}_{E s t}=\{0\}
\end{gathered}
$$

Here, $\left\{\frac{\partial P 1}{\partial z}\right\}$ and $\left\{\frac{\partial P N}{\partial z}\right\}$ are column vectors containing the axial derivative of the acoustic pressure at the $M$ finite element nodes located along the source and duct termination planes, respectively. The measured values, $\left\{\frac{\partial P 1}{\partial z}\right\}_{\text {Meas }}$, are obtained by differentiating Eqs. (25) and (27), whereas the finite element solutions, $\left\{\frac{\partial P 1}{\partial z}\right\}_{\text {Est }}$, are obtained from the solution to Eq. (22). Thus Eq. (22) is solved to obtain the axial derivative of the acoustic pressure in the duct for 
specified wall impedance functions, $\zeta_{1}(z)$ and $\zeta_{1}(z)$, and these impedance functions are iterated upon until the finite element solution for the axial derivative of the acoustic pressure at the source and duct termination planes match the measurement.

The impedance eduction method is now formulated as an optimization problem. That is, values of the impedance coefficients are sought that minimize the objective function

$$
\begin{gathered}
F_{\text {wall }}\left(\zeta_{1}, \zeta_{2}\right)=\left\|\{R\}_{\text {hard }}\right\|_{L 2} \\
\left\{R_{\text {hard }}\right\}=\left\{\begin{array}{l}
\left\{\frac{\partial P 1}{\partial z}\right\}_{\text {Meas }}-\left\{\frac{\partial P 1}{\partial z}\right\}_{E s t} \\
\left\{\frac{\partial P N}{\partial z}\right\}_{\text {Meas }}-\left\{\frac{\partial P 1}{\partial z}\right\}_{\text {Est }}
\end{array}\right\}
\end{gathered}
$$

Note that the residual error vector, $R_{\text {hard }}$, is defined as the difference between the measured axial derivative of the acoustic pressure and that computed by the finite element method. Further, using arguments identical to those in the previous section, it is easily shown that the wall impedance functions that minimize $F_{\text {wall }}\left(\zeta_{1}, \zeta_{2}\right)$ are the unknown impedance functions of the test liners.

\section{Results and Discussion}

\section{A. The Computer Code}

An in-house computer code (MMFEM) that implements the impedance eduction methodologies discussed in this paper has been developed. The code uses Fortran 90 with double precision (i.e., 64 bit) arithmetic and is designed to run in a shared memory environment. This approach is chosen because the primary hardware to be utilized is an Altix 350 computer system. In the current implementation, the resistance, $\theta$, and reactance, $\chi$, of the test liner are the design variables $\left(\zeta_{1}=\theta_{1}+i \chi_{1}\right.$ and $\left.\zeta_{2}=\theta_{2}+i \chi_{2}\right)$ for softwall configurations. On the other hand, the conductance, $\Re\left[1 / \zeta_{2}\right]$, and susceptance, $\mathfrak{I}\left[1 / \zeta_{2}\right]$, are the design variables for hardwall configurations, where $\mathfrak{R}[]$ and $\mathfrak{I}[]$ denote the real and imaginary parts, respectively, of the complex quantity within the brackets. In addition, each set of results is restricted to a uniform liner on the upper wall with a rigid lower wall $\left(\zeta_{2}=\right.$ constant, and $\left.1 / \zeta_{1}=0+0 i\right)$. Thus, only two design variables $\left(\theta_{2}\right.$ and $\left.\chi_{2}\right)$ are allowed to vary in the optimization algorithm. Each linear system [Eq. (22)] is solved using Pardiso $^{21}$ (a state-of-the-art parallel sparse solver with equation reordering and iterative refinement).

One of the most important aspects of the impedance eduction technique is the optimization algorithm. The optimization algorithm chosen is Stewart's adaptation of the Davidon-Fletcher-Powell (SDFP) optimization algorithm that uses a finite difference approximation to the gradient of the objective function. ${ }^{22}$ This gradient-based optimizer has the disadvantage that it may converge to local optima (if they exist), and may become stuck in that portion of the impedance plane where the objective function is extremely flat. However, the above shortcomings of SDFP are more than mitigated by the fact that SDFP tends to converge faster and give more accurate results than many of its competitors. Further, the occurrence of multiple local optima has to date not been observed with the impedance eduction methodologies. However, the authors are not aware of any definitive proof that multiple local optima cannot exist in any of the impedance eduction procedures. Consequently, multiple initial starting values for the impedance coefficients are used to strengthen our confidence that the global optimum is achieved. Recently, a global-based optimizer, the "Genetic Algorithm," was added to our suite of optimization tools. Although the Genetic Algorithm ${ }^{23}$ runs in parallel and locates a global optimum, it tends to be much more computationally expensive because it performs a global search in the complex impedance plane. For this reason, the Genetic Algorithm was used only sparingly in this paper, and then only to spot-check the integrity of impedances educed using SDFP. In all cases, no noticeable differences between the impedance educed using SDFP and the Genetic Algorithm were observed. Therefore, only the SDFP results are presented in this section. The optimizer (i.e., SDFP) runs only in sequential mode, using central finite differences to compute the gradient of the objective function, and the algorithm is currently unconstrained. Unless otherwise stated, a stopping criteria (i.e., tolerance) of $1 \times 10^{-8}$ is used (i.e., $F\left(\zeta_{1}, \zeta_{2}\right) \leq 1 \times 10^{-8}$ ) to terminate the search for each test liner.

\section{B. Duct Geometry and Mean Flow Field}

Results in this section are restricted to that of the geometry of the CDTR (i.e., $L_{1}=0.203 \mathrm{~m}, L_{2}=1.016 \mathrm{~m}, L=$ $1.219 \mathrm{~m}, W=0.381 \mathrm{~m}$ and $H=0.152 \mathrm{~m})$. Thus, as many as seven horizontal modes $(\bar{M}=7)$ may propagate without axial decay for the lowest order horizontal mode (i.e., $n=0)$. Results are obtained for six flow Mach numbers $\left(M_{0}=0.0\right.$, $0.1,0.2,0.3,0.4$, and 0.5 ) and all results are computed at standard atmospheric conditions, so that $T_{0}=295.00 \mathrm{~K}$, $c_{0}=344.28 \mathrm{~m} / \mathrm{s}, \rho_{0}=1.20 \mathrm{~kg} / \mathrm{m}^{3}$, and $P_{0}=101325.00 \mathrm{~Pa}$. 


\section{Hardwall Results}

Several examples are presented to demonstrate the accuracy and robustness of the impedance eduction methodologies in a hardwall duct. In these examples, the normalized conductance $\left(\Re\left[1 / \zeta_{1}\right]\right)$ and susceptance $\left(\mathfrak{I}\left[1 / \zeta_{1}\right]\right)$ of the lower wall are set to a zero value and the normalized conductance $\left(\mathfrak{R}\left[1 / \zeta_{2}\right]\right)$ and susceptance $\left(\mathfrak{I}\left[1 / \zeta_{2}\right]\right)$ of the the upper wall are educed. The CDTR is not currently configured to satisfy configuration 2 because the microphone array on the adjacent wall has not yet been installed. The data required for these hardwall examples are therefore synthesized from the exact mode solution for outgoing waves in an infinite hardwall duct:

$$
p(z, x, y)=\sum_{m=0}^{\bar{M}-1} \sum_{n=0}^{\bar{N}-1} A_{m, n}^{+} e^{i K_{m, n}^{+} z^{z}} \cos \left(\frac{n \pi x}{H}\right) \cos \left(\frac{m \pi y}{W}\right)
$$

Equation (32) is used to extract the source and duct termination pressures that are required to construct the finite element matrix equation [Eq. (22)]. Equation (32) is also used to construct the microphone pressures needed for impedance eduction in configurations 1 and 2, and the pressure gradients needed in configuration 3 . Further, only the modes that do not attenuate are allowed to propagate through the duct and these are given an amplitude of $20 \mathrm{~Pa}$ and zero phase

$$
A_{m, n}^{+}=20 \mathrm{~Pa}
$$

The amplitude and phase of each attenuating mode is given a zero value $\left(A_{m, n}^{+}=0.0\right)$.

A uniform spatial grid with 97 gridlines in the axial direction $(N=97)$ and 13 gridlines in the vertical direction $(M=13)$ is used to educe the impedance from the synthesized data. The uniform grid contains 65 equally spaced axial points in the liner region and all 65 points are used to construct the microphone array. The microphone array is a line array (i.e., it is located only along a single horizontal gridline). The normalized conductance and susceptance are educed for each of the six Mach numbers at six selected frequencies $(f=0.5,1.0,1.5,2.0,2.5$, and $3.0 \mathrm{kHz})$ using the planar source. Although results are not given, educed normalized conductance and susceptance values for each of the three configurations in Fig. 1 are in excellent agreement with the expected value of zero to four decimal digits of precision.

Table 1 shows results for Mach 0.0-0.5 when the sound source contains only the first order vertical mode (i.e., the $(0,1)$ mode). The first and second columns in the table contain the frequency, $f$, in $\mathrm{kHz}$ and the uniform flow Mach number, $M_{0}$, respectively. The lowest frequency in the table $(1.5 \mathrm{kHz})$ is the smallest frequency for which the $(0,1)$ mode does not attenuate. To highlight the effects of the placement of microphones at pressure nulls, table 1 presents results for two microphone arrays in configuration 2. The first microphone array was a line array located at the vertical location, $x=H / 4$, and these results are tabulated in columns 5 and 6 . The second microphone array was a line array located at the vertical location, $x=H / 2$ (the location of acoustic pressure nulls), and these results are tabulated in columns 7 and 8 . Note that when the microphones are located at pressure nulls (i.e., $x=H / 2$ for the $(0,1)$ mode), significant errors in the educed normalized susceptance occur. However, when the microphone array is located away from pressure nulls (i.e., $x=H / 4$ ), normalized conductance and susceptance values educed using the array of microphones adjacent to the test liner compare quite favorably to the expected value of zero. Also, normalized conductance and susceptance values educed using configurations 1 and 3 (for which the microphone data used does not contain pressure nulls) are in excellent agreement with their expected value of zero.

Table 2 show results similar to those in table 1 but for a sound source containing equal mode amplitudes and zero phases in the first three hardwall duct modes $\left(A_{0,0}=A_{0,1}=A_{0,2}=20 \mathrm{~Pa}\right)$. The impedance eduction methodology for configuration 2 is observed to be more forgiving to having microphones along the null line of the $(0,1)$ mode than was observed when the source contains only the $(0,1)$ mode. However, improvements in the accuracy of the educed normalized conductance and susceptance are clearly observed in configuration 2 when the microphones are placed away from locations where pressure nulls occur.

\section{Softwall Results}

To test the ability of the impedance eduction methodologies to accurately educe the impedance for a softwall, a liner consisting of a perforated plate over honeycomb is installed into the GIT, and its impedance is educed (using measured data from the GIT) for several flow Mach numbers. Next, the normalized impedance for the liner is educed using the geometry of the CDTR. Because the necessary input data is not available from CDTR measurements, the

quantities required to educe the impedance (i.e., $p_{\text {Meas }}\left(z_{I}\right)$ for configurations 1 and $2 ;\left\{\frac{\partial P 1}{\partial z}\right\}_{\text {Meas }}$ and $\left\{\frac{\partial P N}{\partial z}\right\}_{E s t}$ for configuration 3) are simulated by running the finite-element program with the liner impedance set to that educed in the 
Table 1. Educed normalized conductance, $\Re\left[1 / \zeta_{2}\right]$, and normalized susceptance, $\mathfrak{I}\left[1 / \zeta_{2}\right]$, of hardwall duct with the $(0,1)$ mode source.

\begin{tabular}{|r|r|c|c|c|c|c|c|c|c|}
\hline & & \multicolumn{2}{|c|}{ Configuration 1 } & \multicolumn{2}{c|}{$\begin{array}{c}\text { Configuration 2 } \\
\text { array at, } x=H / 4\end{array}$} & \multicolumn{2}{c|}{$\begin{array}{c}\text { Configuration } 2 \\
\text { array at, } x=H / 2\end{array}$} & \multicolumn{2}{c|}{ Configuration 3} \\
\hline$f$ & $M_{0}$ & $\Re\left[1 / \zeta_{2}\right]$ & $\mathfrak{I}\left[1 / \zeta_{2}\right]$ & $\Re\left[1 / \zeta_{2}\right]$ & $\mathfrak{I}\left[1 / \zeta_{2}\right]$ & $\Re\left[1 / \zeta_{2}\right]$ & $\mathfrak{I}\left[1 / \zeta_{2}\right]$ & $\Re\left[1 / \zeta_{2}\right]$ & $\mathfrak{I}\left[1 / \zeta_{2}\right]$ \\
\hline 1.5 & 0 & 0.0000 & 0.0000 & 0.0000 & 0.0026 & 0.0000 & 0.2361 & 0.0000 & 0.0000 \\
\hline 2 & 0 & 0.0000 & 0.0000 & 0.0000 & 0.0270 & 0.0000 & 0.0985 & 0.0000 & 0.0000 \\
\hline 2.5 & 0 & 0.0000 & 0.0000 & 0.0000 & 0.0219 & 0.0000 & 0.0739 & 0.0000 & 0.0000 \\
\hline 3 & 0 & 0.0000 & 0.0000 & 0.0000 & 0.0293 & 0.0001 & 0.1060 & 0.0000 & 0.0000 \\
\hline 1.5 & 0.1 & 0.0000 & 0.0000 & 0.0000 & 0.0066 & 0.0000 & 0.1569 & 0.0000 & 0.0000 \\
\hline 2 & 0.1 & 0.0000 & 0.0000 & 0.0000 & 0.0191 & 0.0000 & 0.0569 & 0.0000 & 0.0000 \\
\hline 2.5 & 0.1 & 0.0000 & 0.0000 & 0.0000 & 0.0062 & 0.0000 & 0.1710 & 0.0000 & 0.0000 \\
\hline 3 & 0.1 & 0.0000 & 0.0000 & 0.0000 & 0.0156 & 0.0000 & 0.0444 & 0.0000 & 0.0000 \\
\hline 1.5 & 0.2 & 0.0000 & 0.0000 & 0.0000 & 0.0257 & 0.0000 & 0.1184 & 0.0000 & 0.0000 \\
\hline 2 & 0.2 & 0.0000 & 0.0000 & 0.0000 & 0.0287 & 0.0000 & 0.1779 & 0.0000 & 0.0000 \\
\hline 2.5 & 0.2 & 0.0000 & 0.0000 & 0.0000 & 0.0446 & 0.0017 & 0.1271 & 0.0000 & 0.0000 \\
\hline 3 & 0.2 & 0.0000 & 0.0000 & 0.0000 & 0.0432 & 0.0000 & 0.1160 & 0.0000 & 0.0000 \\
\hline 1.5 & 0.3 & 0.0000 & 0.0000 & 0.0000 & 0.0084 & 0.0000 & 0.1211 & 0.0000 & 0.0000 \\
\hline 2 & 0.3 & 0.0000 & 0.0000 & 0.0010 & 0.0077 & 0.0198 & 0.1902 & 0.0000 & 0.0000 \\
\hline 2.5 & 0.3 & 0.0000 & 0.0000 & 0.0000 & 0.0492 & 0.0000 & 0.1031 & 0.0000 & 0.0000 \\
\hline 3 & 0.3 & 0.0000 & 0.0000 & 0.0000 & 0.0295 & 0.0000 & 0.0554 & 0.0000 & 0.0000 \\
\hline 1.5 & 0.4 & 0.0000 & 0.0000 & 0.0000 & 0.0109 & 0.0000 & 0.0986 & 0.0000 & 0.0000 \\
\hline 2 & 0.4 & 0.0000 & 0.0000 & 0.0000 & 0.0455 & 0.0000 & 0.1037 & 0.0000 & 0.0000 \\
\hline 2.5 & 0.4 & 0.0000 & 0.0000 & 0.0009 & 0.0173 & 0.0000 & 0.1280 & 0.0000 & 0.0000 \\
\hline 3 & 0.4 & 0.0000 & 0.0000 & 0.0000 & 0.0337 & 0.0000 & 0.0501 & 0.0000 & 0.0000 \\
\hline 1 & 0.5 & 0.0000 & 0.0000 & 0.0000 & 0.0017 & 0.0000 & 0.0568 & 0.0000 & 0.0000 \\
\hline 1.5 & 0.5 & 0.0000 & 0.0000 & 0.0000 & 0.0221 & 0.0000 & 0.1539 & 0.0000 & 0.0000 \\
\hline 2 & 0.5 & 0.0000 & 0.0000 & 0.0000 & 0.0469 & 0.0000 & 0.0740 & 0.0000 & 0.0000 \\
\hline 2.5 & 0.5 & 0.0000 & 0.0000 & 0.0001 & 0.0315 & 0.0441 & 0.1782 & 0.0000 & 0.0000 \\
\hline 3 & 0.5 & 0.0000 & 0.0000 & 0.0000 & 0.0268 & 0.0761 & 0.1288 & 0.0000 & 0.0000 \\
\hline
\end{tabular}


Table 2. Educed normalized conductance, $\Re\left[1 / \zeta_{2}\right]$, and normalized susceptance, $\mathfrak{I}\left[1 / \zeta_{2}\right]$, of hardwall duct with an equal mode amplitude source.

\begin{tabular}{|c|c|c|c|c|c|c|c|c|c|}
\hline & & \multicolumn{2}{|c|}{ Configuration 1} & \multicolumn{2}{|c|}{$\begin{array}{c}\text { Configuration } 2 \\
\text { array at, } x=H / 4\end{array}$} & \multicolumn{2}{|c|}{$\begin{array}{c}\text { Configuration } 2 \\
\text { array at, } x=H / 2\end{array}$} & \multicolumn{2}{|c|}{ Configuration 3} \\
\hline$f$ & $M_{0}$ & $\Re\left[1 / \zeta_{2}\right]$ & $\mathfrak{I}\left[1 / \zeta_{2}\right]$ & $\Re\left[1 / \zeta_{2}\right]$ & $\mathfrak{I}\left[1 / \zeta_{2}\right]$ & $\Re\left[1 / \zeta_{2}\right]$ & $\mathfrak{I}\left[1 / \zeta_{2}\right]$ & $\Re\left[1 / \zeta_{2}\right]$ & $\mathfrak{I}\left[1 / \zeta_{2}\right]$ \\
\hline 0.5 & 0 & 0.0000 & 0.0000 & 0.0000 & 0.0000 & 0.0000 & 0.0000 & 0.0000 & 0.0000 \\
\hline 1 & 0 & 0.0000 & 0.0000 & 0.0000 & 0.0000 & 0.0000 & 0.0000 & 0.0000 & 0.0000 \\
\hline 1.5 & 0 & 0.0000 & 0.0000 & 0.0001 & 0.0025 & 0.0160 & 0.0546 & 0.0000 & 0.0000 \\
\hline 2 & 0 & 0.0000 & 0.0000 & 0.0006 & 0.0169 & 0.0004 & 0.0988 & 0.0000 & 0.0000 \\
\hline 2.5 & 0 & 0.0000 & 0.0000 & 0.0014 & 0.0264 & 0.1918 & 0.1033 & 0.0000 & 0.0000 \\
\hline 3 & 0 & 0.0000 & 0.0000 & 0.0108 & 0.0197 & 0.0028 & 0.0400 & 0.0000 & 0.0000 \\
\hline 0.5 & 0.1 & 0.0000 & 0.0000 & 0.0000 & 0.0000 & 0.0000 & 0.0000 & 0.0000 & 0.0000 \\
\hline 1 & 0.1 & 0.0000 & 0.0000 & 0.0000 & 0.0000 & 0.0000 & 0.0000 & 0.0000 & 0.0000 \\
\hline 1.5 & 0.1 & 0.0000 & 0.0000 & 0.0001 & -0.0063 & 0.0073 & 0.0377 & 0.0000 & 0.0000 \\
\hline 2 & 0.1 & 0.0000 & 0.0000 & 0.0008 & 0.0197 & 0.0002 & 0.0572 & 0.0000 & 0.0000 \\
\hline 2.5 & 0.1 & 0.0000 & 0.0000 & 0.0002 & 0.0068 & 0.1889 & -0.5678 & 0.0000 & 0.0000 \\
\hline 3 & 0.1 & 0.0000 & 0.0000 & 0.0018 & 0.0131 & 0.2491 & 0.1393 & 0.0000 & 0.0000 \\
\hline 0.5 & 0.2 & 0.0000 & 0.0000 & 0.0000 & 0.0000 & 0.0000 & 0.0000 & 0.0000 & 0.0000 \\
\hline 1 & 0.2 & 0.0000 & 0.0000 & 0.0000 & 0.0000 & 0.0000 & 0.0000 & 0.0000 & 0.0000 \\
\hline 1.5 & 0.2 & 0.0000 & 0.0000 & 0.0012 & 0.0021 & 0.0007 & 0.1197 & 0.0000 & 0.0000 \\
\hline 2 & 0.2 & 0.0000 & 0.0000 & 0.0035 & -0.0280 & 0.0325 & 0.0756 & 0.0000 & 0.0000 \\
\hline 2.5 & 0.2 & 0.0000 & & 0.0000 & & & & 0.0000 & 0.0000 \\
\hline 3 & 0.2 & 0.0000 & 0.0000 & 0.0000 & 0.0263 & 0.6794 & -0.2020 & 0.0000 & 0.0000 \\
\hline 0.5 & 0.3 & 0.0000 & 0.0000 & 0.0000 & 0.0000 & 0.0000 & 0.0000 & 0.0000 & 0.0000 \\
\hline 1 & 0.3 & 0.0000 & 0.0000 & 0.0000 & 0.0000 & 0.0000 & 0.0000 & 0.0000 & 0.0000 \\
\hline 1.5 & 0.3 & 0.0000 & 0.0000 & 0.0001 & -0.0081 & 0.0040 & 0.0581 & 0.0000 & 0.0000 \\
\hline 2 & 0.3 & 0.0000 & 0.0000 & 0.0007 & -0.0102 & 0.0331 & 0.1235 & 0.0000 & 0.0000 \\
\hline 2.5 & 0.3 & 0.0000 & 0.0000 & 0.0000 & 0.0314 & 0.2193 & 0.1285 & 0.0000 & 0.0000 \\
\hline 3 & 0.3 & 0.0000 & 0.0000 & 0.0029 & 0.0244 & 0.0014 & 0.3623 & 0.0000 & 0.0000 \\
\hline 0.5 & 0.4 & 0.0000 & 0.0000 & 0.0000 & 0.0000 & 0.0000 & 0.0000 & 0.0000 & 0.0000 \\
\hline 1 & 0.4 & 0.0000 & 0.0000 & 0.0000 & 0.0000 & 0.0000 & 0.0000 & 0.0000 & 0.0000 \\
\hline 1.5 & 0.4 & 0.0000 & 0.0000 & 0.0002 & -0.0106 & 0.0120 & 0.1308 & 0.0000 & 0.0000 \\
\hline 2 & 0.4 & 0.0000 & 0.0000 & 0.0056 & 0.0208 & 0.0005 & 0.1076 & 0.0000 & 0.0000 \\
\hline 2.5 & 0.4 & 0.0000 & 0.0000 & 0.0057 & -0.0015 & 0.4006 & -1.3447 & 0.0000 & 0.0000 \\
\hline 3 & 0.4 & 0.0000 & 0.0000 & 0.0010 & 0.0281 & 0.2100 & 0.0210 & 0.0000 & 0.0000 \\
\hline 0.5 & 0.5 & 0.0000 & 0.0000 & 0.0000 & 0.0000 & 0.0000 & 0.0000 & 0.0000 & 0.0000 \\
\hline 1 & 0.5 & 0.0000 & 0.0000 & 0.0000 & 0.0017 & 0.0001 & 0.0569 & 0.0000 & 0.0000 \\
\hline 1.5 & 0.5 & 0.0000 & 0.0000 & 0.0010 & 0.0217 & 0.0097 & 0.1565 & 0.0000 & 0.0000 \\
\hline 2 & 0.5 & 0.0000 & 0.0000 & 0.0001 & 0.0594 & 0.1978 & 0.1545 & 0.0000 & 0.0000 \\
\hline 2.5 & 0.5 & 0.0000 & 0.0000 & 0.0031 & 0.0326 & 0.1956 & 0.1738 & 0.0000 & 0.0000 \\
\hline 3 & 0.5 & 0.0000 & 0.0000 & 0.0024 & 0.0238 & 0.2219 & 0.0304 & 0.0000 & 0.0000 \\
\hline
\end{tabular}


GIT. The source and termination plane acoustic pressure profiles are identical to those in the hardwall example. The spatial grid and location of the microphones used in the softwall simulations are identical to that used in the hardwall example.

Table 3. Comparison of the educed normalized resistance, $\theta_{2}$, and normalized reactance, $\chi_{2}$, of test liner with a plane wave source.

\begin{tabular}{|r|c|c|c|c|c|c|c|c|c|}
\hline & & \multicolumn{2}{|c|}{ GIT } & \multicolumn{2}{c|}{ Configuration 1 } & \multicolumn{2}{c|}{$\begin{array}{c}\text { Configuration 2 } \\
\text { array at, } x=H / 2\end{array}$} & \multicolumn{2}{c|}{ Configuration 3 } \\
\hline$f$ & $M_{0}$ & $\theta_{2}$ & $\chi_{2}$ & $\theta_{2}$ & $\chi_{2}$ & $\theta_{2}$ & $\chi_{2}$ & $\theta_{2}$ & $\chi_{2}$ \\
\hline 0.5 & 0.0 & 0.5347 & -2.7893 & 0.5347 & -2.7893 & 0.5347 & -2.7893 & 0.5347 & -2.7893 \\
\hline 1.0 & 0.0 & 0.1984 & -1.1681 & 0.1984 & -1.1681 & 0.1984 & -1.1681 & 0.1984 & -1.1681 \\
\hline 1.5 & 0.0 & 0.2282 & -0.1371 & 0.2282 & -0.1371 & 0.2282 & -0.1371 & 0.2282 & -0.1371 \\
\hline 2.0 & 0.0 & 0.1150 & 0.3469 & 0.1150 & 0.3469 & 0.1150 & 0.3469 & 0.1150 & 0.3469 \\
\hline 2.5 & 0.0 & 0.1598 & 0.6945 & 0.1598 & 0.6945 & 0.1598 & 0.6945 & 0.1598 & 0.6945 \\
\hline 0.5 & 0.3 & 1.7279 & -1.7588 & 1.7279 & -1.7588 & 1.7279 & -1.7588 & 1.7279 & -1.7588 \\
\hline 1.0 & 0.3 & 0.8210 & -0.8741 & 0.8210 & -0.8741 & 0.8210 & -0.8741 & 0.8210 & -0.8741 \\
\hline 1.5 & 0.3 & 0.7517 & -0.3958 & 0.7517 & -0.3958 & 0.7517 & -0.3958 & 0.7517 & -0.3958 \\
\hline 2.0 & 0.3 & 0.7340 & 0.1476 & 0.7340 & 0.1476 & 0.7340 & 0.1476 & 0.7340 & 0.1476 \\
\hline 2.5 & 0.3 & 0.8323 & 0.4356 & 0.8323 & 0.4356 & 0.8323 & 0.4356 & 0.8323 & 0.4356 \\
\hline
\end{tabular}

Table 4. Comparison of the educed normalized resistance, $\theta_{2}$, and normalized reactance, $\chi_{2}$ of test liner with the $(0,1)$ mode source.

\begin{tabular}{|r|c|c|c|c|c|c|c|c|c|}
\hline & & \multicolumn{2}{|c|}{ GIT } & \multicolumn{2}{c|}{ Configuration 1 } & \multicolumn{2}{c|}{$\begin{array}{c}\text { Configuration 2 } \\
\text { array at, } x=H / 2\end{array}$} & \multicolumn{2}{c|}{ Configuration 3 } \\
\hline$f$ & $M_{0}$ & $\theta_{2}$ & $\chi_{2}$ & $\theta_{2}$ & $\chi_{2}$ & $\theta_{2}$ & $\chi_{2}$ & $\theta_{2}$ & $\chi_{2}$ \\
\hline 0.5 & 0.0 & 0.5347 & -2.7893 & 0.5347 & -2.7893 & 0.5347 & -2.7893 & 0.5347 & -2.7893 \\
\hline 1.0 & 0.0 & 0.1984 & -1.1681 & 0.1984 & -1.1681 & 0.1984 & -1.1681 & 0.1984 & -1.1681 \\
\hline 1.5 & 0.0 & 0.2282 & -0.1371 & 0.2282 & -0.1371 & 0.2282 & -0.1371 & 0.2282 & -0.1371 \\
\hline 2.0 & 0.0 & 0.1150 & 0.3469 & 0.1150 & 0.3469 & 0.1150 & 0.3469 & 0.1150 & 0.3469 \\
\hline 2.5 & 0.0 & 0.1598 & 0.6945 & 0.1598 & 0.6945 & 0.1598 & 0.6945 & 0.1598 & 0.6945 \\
\hline 0.5 & 0.3 & 1.7279 & -1.7588 & 1.7279 & -1.7588 & 1.7279 & -1.7588 & 1.7279 & -1.7588 \\
\hline 1.0 & 0.3 & 0.8210 & -0.8741 & 0.8210 & -0.8741 & 0.8210 & -0.8741 & 0.8210 & -0.8741 \\
\hline 1.5 & 0.3 & 0.7517 & -0.3958 & 0.7517 & -0.3958 & 0.7517 & -0.3958 & 0.7517 & -0.3958 \\
\hline 2.0 & 0.3 & 0.7340 & 0.1476 & 0.7340 & 0.1476 & 0.7340 & 0.1476 & 0.7340 & 0.1476 \\
\hline 2.5 & 0.3 & 0.8323 & 0.4356 & 0.8323 & 0.4356 & 0.8323 & 0.4356 & 0.8323 & 0.4356 \\
\hline
\end{tabular}

Educed normalized resistance and susceptance values for each of the three duct configurations (see Fig. 1) using the CDTR geometry are compared to that measured in the GIT for the plane wave source, the $(0,1)$ mode source, and the equal mode amplitude source in tables 3-5, respectively. Results for duct configuration 2 are presented when the array of microphones is located along the vertical gridline, $x=H / 2$ (identical results were obtained when the vertical position of the microphone array was located at $x=H / 4$ ). The educed impedance for each of the three duct configurations is in agreement with values obtained in the GIT to five significant digits for the planar source (table 3), the $(0,1)$ mode source (table 4$)$, and the equal mode amplitude source (table 5). Note that the acoustic pressure nulls that are located on the right sidewall at $x=H / 2$ in the hardwall duct example are no longer present in this softwall example. This occurs because of scattering of acoustic energy out of the hardwall duct modes into softwall duct modes at the leading and trailing edges of the liner. The softwall duct modes do not have pressure nulls along the right sidewall. Consequently, the loss of accuracy in educed impedance that was encountered for configuration 2 in the hardwall example is not experienced when a liner is present. 
Table 5. Comparison of the educed normalized resistance, $\theta_{2}$, and normalized reactance, $\chi_{2}$, of test liner with an equal mode amplitude source.

\begin{tabular}{|r|c|c|c|c|c|c|c|c|c|}
\hline & & \multicolumn{2}{|c|}{ GIT } & \multicolumn{2}{c|}{ Configuration 1 } & \multicolumn{2}{c|}{$\begin{array}{c}\text { Configuration 2 } \\
\text { array at, } x=H / 2\end{array}$} & \multicolumn{2}{c|}{ Configuration 3 } \\
\hline$f$ & $M_{0}$ & $\theta_{2}$ & $\chi_{2}$ & $\theta_{2}$ & $\chi_{2}$ & $\theta_{2}$ & $\chi_{2}$ & $\theta_{2}$ & $\chi_{2}$ \\
\hline 0.5 & 0.0 & 0.5347 & -2.7893 & 0.5347 & -2.7893 & 0.5347 & -2.7893 & 0.5347 & -2.7893 \\
\hline 1.0 & 0.0 & 0.1984 & -1.1681 & 0.1984 & -1.1681 & 0.1984 & -1.1681 & 0.1984 & -1.1681 \\
\hline 1.5 & 0.0 & 0.2282 & -0.1371 & 0.2282 & -0.1371 & 0.2282 & -0.1371 & 0.2282 & -0.1371 \\
\hline 2.0 & 0.0 & 0.1150 & 0.3469 & 0.1150 & 0.3469 & 0.1150 & 0.3469 & 0.1150 & 0.3469 \\
\hline 2.5 & 0.0 & 0.1598 & 0.6945 & 0.1598 & 0.6945 & 0.1598 & 0.6945 & 0.1598 & 0.6945 \\
\hline 0.5 & 0.3 & 1.7279 & -1.7588 & 1.7279 & -1.7588 & 1.7279 & -1.7588 & 1.7279 & -1.7588 \\
\hline 1.0 & 0.3 & 0.8210 & -0.8741 & 0.8210 & -0.8741 & 0.8210 & -0.8741 & 0.8210 & -0.8741 \\
\hline 1.5 & 0.3 & 0.7517 & -0.3958 & 0.7517 & -0.3958 & 0.7517 & -0.3958 & 0.7517 & -0.3958 \\
\hline 2.0 & 0.3 & 0.7340 & 0.1476 & 0.7340 & 0.1476 & 0.7340 & 0.1476 & 0.7340 & 0.1476 \\
\hline 2.5 & 0.3 & 0.8323 & 0.4356 & 0.8323 & 0.4356 & 0.8323 & 0.4356 & 0.8323 & 0.4356 \\
\hline
\end{tabular}

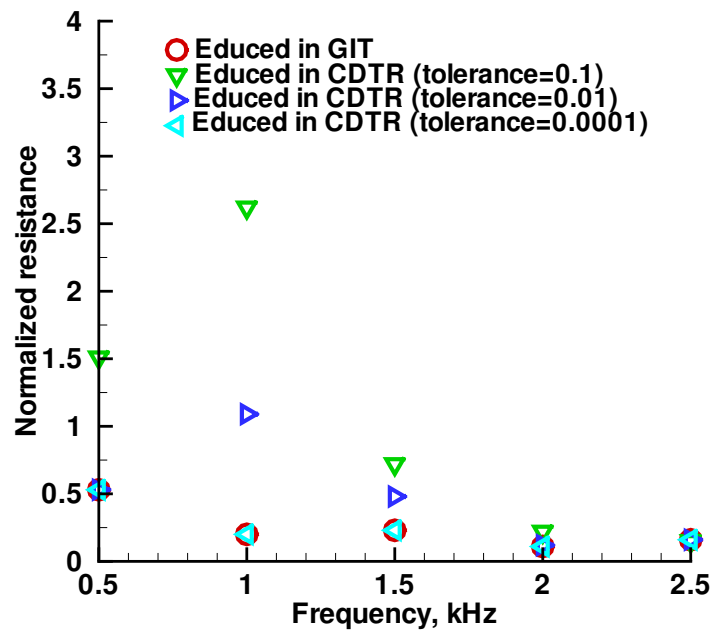

(a) Normalized resistance

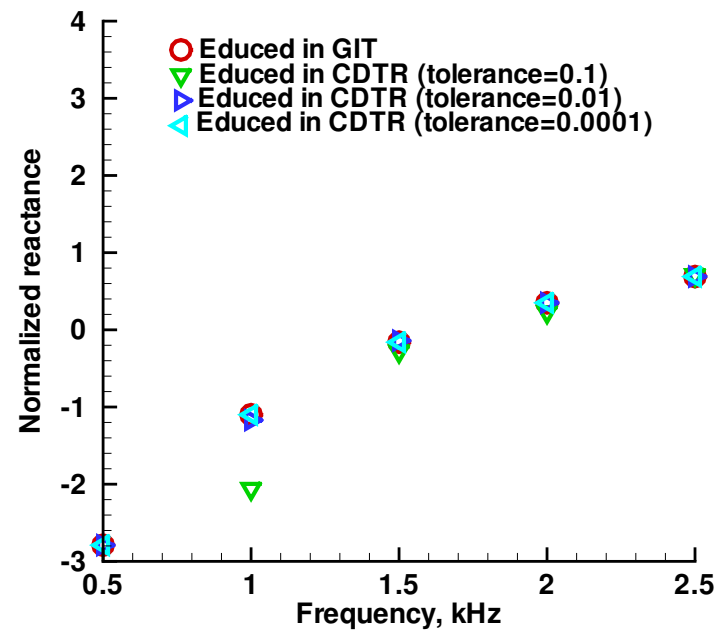

(b) Normalized reactance

Figure 3. Effects of the optimizer tolerance on the educed normalized resistance and reactance of a plane wave source at Mach 0.0. 
It has been observed that the small value of the tolerance used in the computer code (i.e., $10^{-8}$ ) is an important parameter contributing to such excellent agreement between the educed impedance spectrum from the three duct configurations and the spectrum computed in the GIT (see tables 3-5). For example, Fig. 3 compares the educed resistance and reactance for three values of the tolerance $(0.1,0.01$ and 0.0001$)$. The duct configuration depicted in Fig. $1 \mathrm{~b}$ is used, the sound source is a plane wave source, the Mach number is 0.0, and the microphone array lies at the vertical location, $x=H / 2$. It should be noted that a tolerance of 0.1 means that the optimizer terminates the search when the objective function is lower than 0.1 (i.e., $F_{\text {wall }}\left(\zeta_{1}, \zeta_{2}\right) \leq 0.1$ ). Note that a minimum tolerance of 0.0001 is required for accurate impedance eductions. This minimum value of the tolerance (i.e., 0.0001) is also needed for other Mach numbers, sound sources, and duct configurations.

\section{E. Design of a Microphone Array for the CDTR}

Table 6. Selected 32 Microphone Array for the LaRC Curve Duct Test Rig.

\begin{tabular}{|c|c|c|}
\hline Microphone No. & $x$ (in) & $z$ (in) \\
\hline 1 & 1 & 8 \\
2 & 2 & 8 \\
3 & 4 & 8 \\
4 & 5 & 8 \\
5 & 1 & 12 \\
6 & 2 & 12 \\
7 & 4 & 12 \\
8 & 5 & 12 \\
9 & 1 & 16 \\
10 & 2 & 16 \\
11 & 4 & 16 \\
12 & 5 & 16 \\
13 & 1 & 20 \\
14 & 2 & 20 \\
15 & 4 & 20 \\
16 & 5 & 20 \\
\hline
\end{tabular}

\begin{tabular}{|c|c|c|}
\hline Microphone No. & $x$ (in) & $z$ (in) \\
\hline 17 & 1 & 24 \\
18 & 2 & 24 \\
19 & 4 & 24 \\
20 & 5 & 24 \\
21 & 1 & 28 \\
22 & 2 & 28 \\
23 & 4 & 28 \\
24 & 5 & 28 \\
25 & 1 & 32 \\
26 & 2 & 32 \\
27 & 4 & 32 \\
28 & 5 & 32 \\
29 & 1 & 36 \\
30 & 2 & 36 \\
31 & 4 & 36 \\
32 & 5 & 36 \\
\hline
\end{tabular}

Another objective of this work is to design an acceptable microphone array for accurate impedance eduction in the CDTR. A random number generator is used to determine potential locations of 32 microphones on the right sidewall of the CDTR (see Fig. 1b). The number of microphone locations is limited to 32 because this was the number of microphones that are available for insertion into the right sidewall. In addition to the arrays of randomly distributed microphone locations, approximately 100 rectangular grids containing 32 microphones (each located at various locations along the right sidewall) are also investigated. A rectangular array of 32 microphones is determined to be sufficient for the CDTR. Table 6 shows the proposed $x$ and $z$ locations (in inches) for each of the 32 microphones.

Impedances are educed (using the microphone array given in table 6) for the planar wave source, the $(0,1)$ mode source, and the equal mode amplitude sound source. Impedances are educed for Mach 0.0-0.5 in increments of 0.1 and for source frequencies ranging from 0.5 to $3.0 \mathrm{kHz}$ in increments of 0.5 . These represent the ranges of Mach number and frequencies of current interest in the CDTR. Impedances are educed for both hard and softwall duct configurations. The pressure data along the microphone array that is needed for impedance eduction are acquired by running the finite element program using $1 / \zeta_{2}=1 / \zeta_{1}=0$ for the hardwall duct, and with $1 / \zeta_{1}=0$ and the resistance and reactance of $\zeta_{2}$ set to those in the GIT for the softwall duct. These data are then used to educe the liner impedance using the microphone arrangements given in table 6. To account for uncertainty in the input data, the sound pressure levels (SPLs) and phases acquired from running the finite element program are perturbed using a random number generator. The SPL is perturbed by $\pm \frac{1}{2} \mathrm{~dB}$ and the phases by \pm 1 degrees. Such uncertainties in microphone array data $\left( \pm \frac{1}{2} \mathrm{~dB}\right.$, and \pm 1 degrees) are possible for the type of microphones currently under consideration for the CDTR.

Conductance and susceptance values computed for the hardwall duct (i.e., for each of the six Mach numbers and 
all three duct configurations) are in agreement with the exact value of zero to four digits of precision (i.e., with and without random error). These are not shown to limit the number of graphs. Figure 4 compares the educed impedances (i.e., normalized resistance and reactance) with the liner installed to that measured in the GIT using the plane wave source and for Mach 0.0 (first row in Fig. 4) and Mach 0.3. (second row in Fig. 4). The normalized impedance educed using the proposed array is in good agreement with those educed in the GIT. The impact of random errors of $\pm \frac{1}{2} \mathrm{~dB}$ and \pm 1 degrees is small. Figure 5 shows similar results for the equal mode amplitude sound source. The educed normalized impedance using the selected array is again in good agreement with those educed in the GIT. These results confirm that measurements on the wall adjacent to the test liner (the right sidewall in this case) can be used to educe the impedance in the CDTR, with and without higher-order modes, provided the microphone array is properly chosen. Efforts are current underway to implement the proposed microphone array in the CDTR.

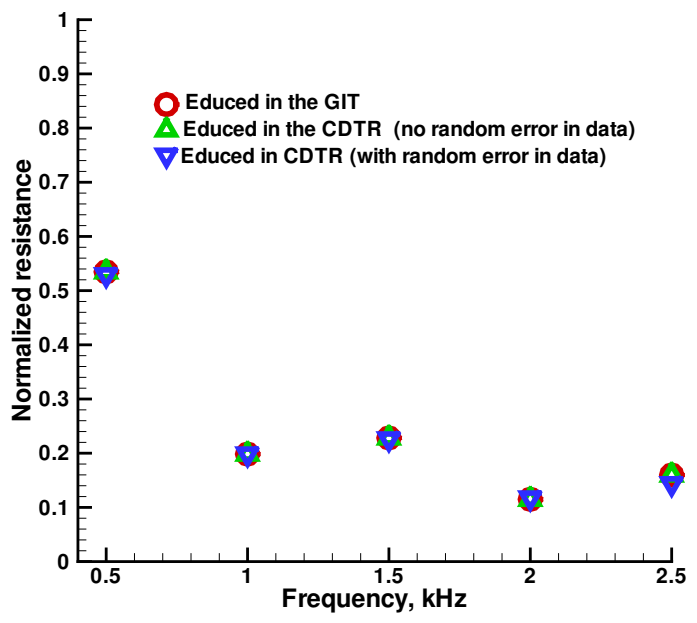

(a) Educed resistance, Mach 0.0

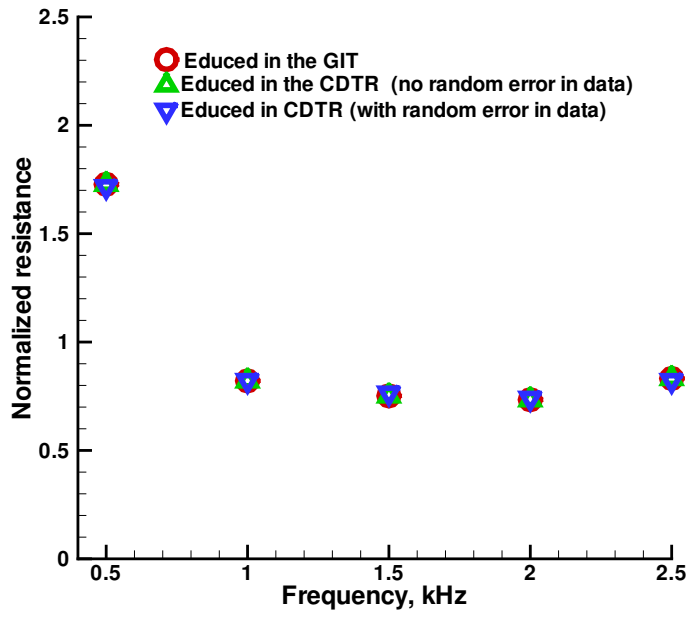

(c) Educed resistance, Mach 0.3

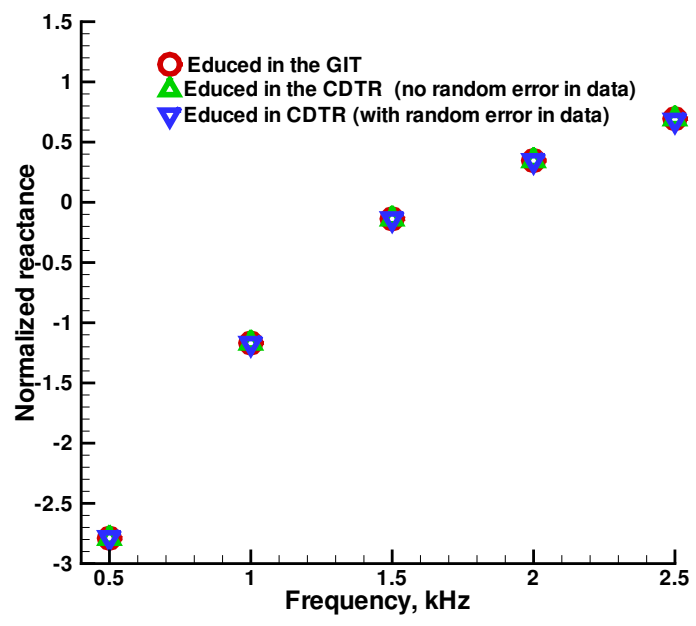

(b) Educed reactance, Mach 0.0

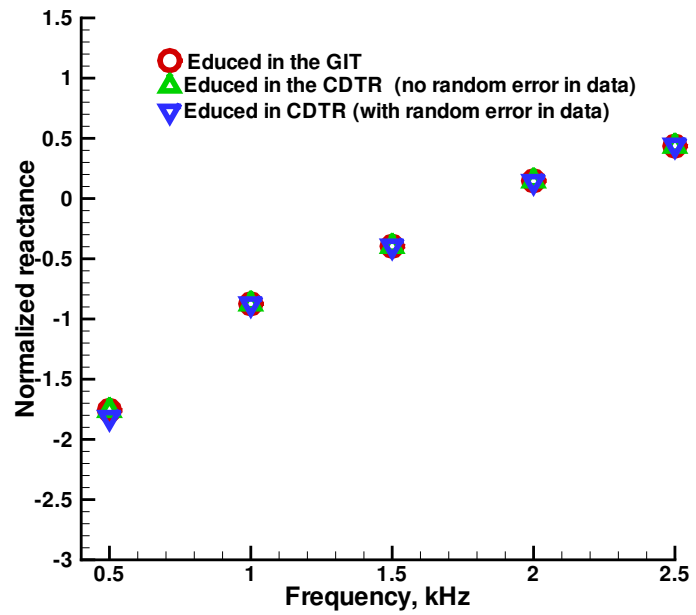

(d) Educed reactance, Mach 0.3

Figure 4. Educed normalized impedance of test liner using the selected array at Mach 0.0 and Mach 0.3 for plane wave source 


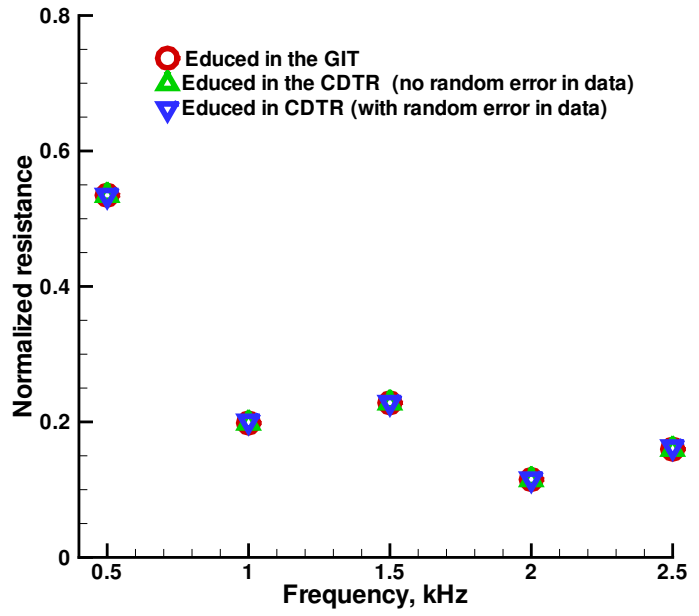

(a) Educed resistance, Mach 0.0

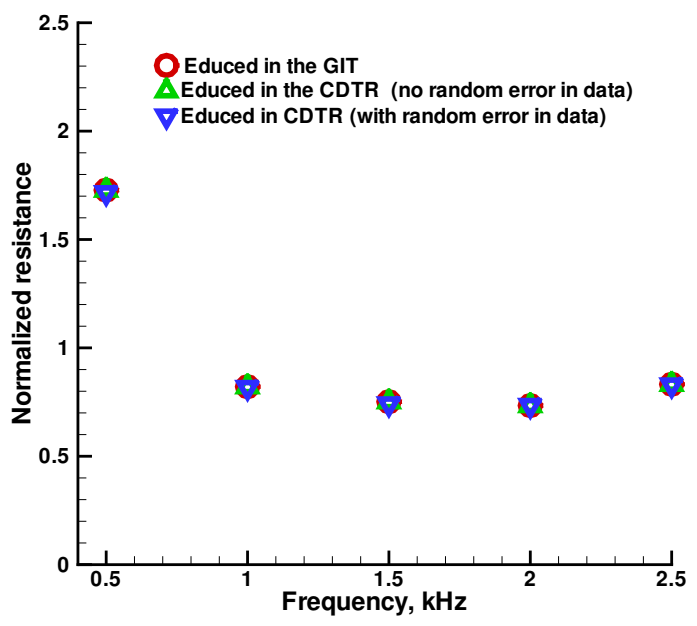

(c) Educed resistance, Mach 0.3

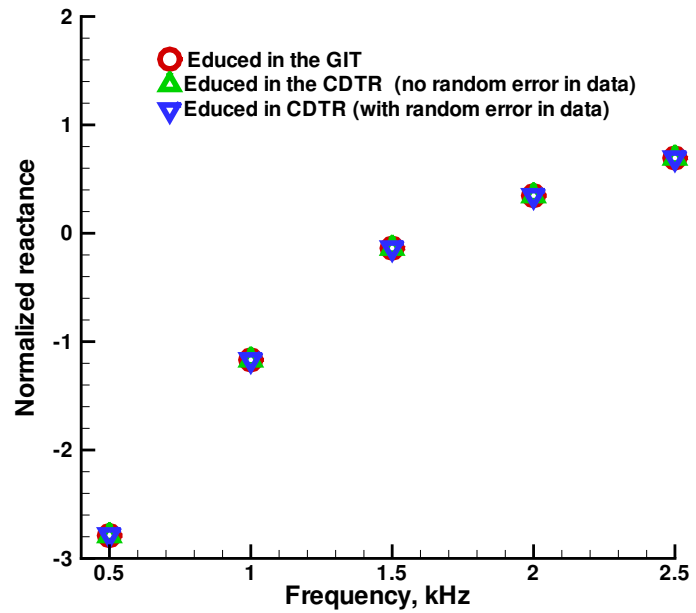

(b) Educed reactance, Mach 0.0

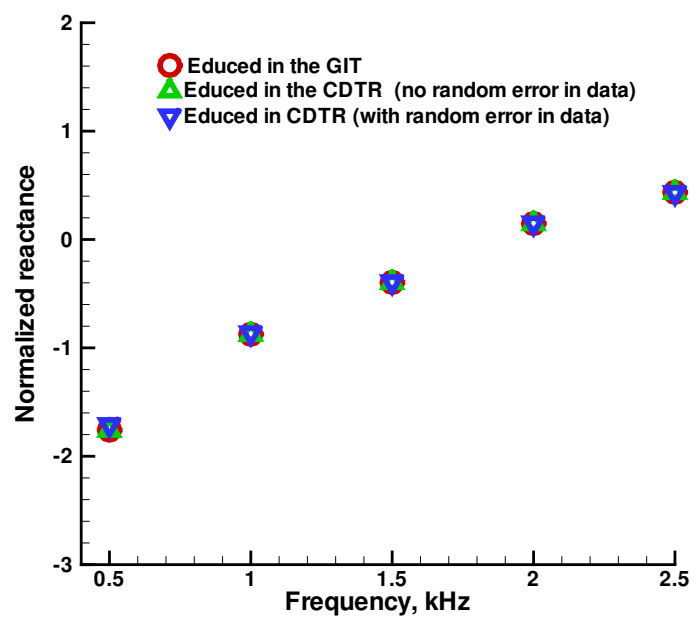

(d) Educed reactance, Mach 0.3

Figure 5. Educed normalized impedance of test liner using the selected array at Mach 0.0 and Mach 0.3 for an equal mode amplitude source 


\section{Conclusions}

An impedance eduction method requiring that a microphone array be imbedded in a wall either opposite or adjacent to the test liner has been successfully extended to include higher order mode effects. A new methodology that eliminates the need for this microphone array has also been presented. Based on the results of this study the following primary conclusions are drawn:

1. The impedance eduction methodology that eliminates the need for a microphone array on a wall either opposite or adjacent to the test liner performs well in the presence of higher order modes and flow.

2. The method requiring that a microphone array be embedded in a wall either opposite or adjacent to the test liner performs well except when most of the microphones are located near acoustic pressure nulls.

3. The value of the tolerance used by the optimizer that educes the impedance is an important factor in both impedance eduction methodologies. Results presented in this paper require a minimum value of $10^{-4}$ for the tolerance.

4. This paper confirms that a rectangular array of 32 microphones, mounted in a wall adjacent to the test liner and with the microphones properly located, may be used to accurately educe the impedance in the presence of higher order modes and mean flow.

\section{References}

\footnotetext{
${ }^{1}$ Armstrong, D. L., Beckemeyer, R. J., and Olsen, R. F., “Impedance Measurements of Acoustic Duct Liners With Grazing Flow,” Paper presented at the 87th Meeting of the Acoustical Society of America, New York, NY, 1974.

${ }^{2}$ Watson W. R., "A New Method for Determining Acoustic-Liner Admittance in Ducts with Sheared Flow in Two Cross-Sectional Directions," NASA TP-2518, 1985.

${ }^{3}$ Watson, W. R., Tanner, S. E., and Parrott, T. L., "Optimization Method for Educing Variable-Impedance Liner Properties," AIAA Journal, Vol. 36, No. 1, 1998, pp. 18-23.

${ }^{4}$ Watson, W. R., Jones, M. G., and Parrott, T. L., "Validation of an Impedance Eduction Method in Flow," AIAA Journal, vol. 37, no. 7, pp. 818-824, July 1999.

${ }^{5}$ Watson, W. R., Tracy, M. B., Jones, M. G., and Parrott, T. L., "Impedance Eduction in the Presence of Shear Flow," AIAA paper 2001-2236, May, 2001.

${ }^{6}$ Jones, M. G., Parrott, T.L., and Watson, W. R., "Comparison of Acoustic Impedance Eduction Techniques for Locally-Reacting Liners," AIAA Paper 2003-3306, May, 2003.

${ }^{7}$ Elnady, T. and Boden, H., "An inverse analytical method for extracting liner impedance from pressure measurements, " AIAA Paper 20042836, May 2004.

${ }^{8}$ Jones, M. G., Watson, W. R., Tracy, M. B., and Parrott, T. L., "Comparison of Two Waveguide Methods for Educing Liner Impedance in Grazing Flow," AIAA Journal, Vol. 42, No. 2, 2004, pp. 232-240.

${ }^{9}$ Auragan, Y., Leroux, M., and Pagneux, V., "Measurement of liner impedance with flow by an inverse method," AIAA Paper 2004-2838, May 2004.

${ }^{10}$ Watson, W. R., Jones, M. G., and Parrott, T. L., "A Quasi-3-D Theory for Impedance Eduction in Uniform Grazing Flow," AIAA Paper 2005-2848, May, 2005.

${ }^{11}$ Watson, W. R., Jones, M. G., and Parrott, T. L., "Comparison of a Convected Helmholtz and Euler Model for Impedance Eduction in Flow, " AIAA paper 2006-2643, May 2006

${ }^{12}$ Elnady, T., Musharraf, M., Boden, H, and Elhadidi, B, "Validation of an Inverse Analytical Technique to Educe Liner Impedance with Grazing Flow," AIAA paper 2006-2639 , May 2006

${ }^{13}$ Watson, W., Jones, M., and Parrott, T., "Investigation of an Anomaly Observed in Impedance Eduction Techniques," AIAA paper 2008-3013, May 2008

${ }^{14}$ Jing, X., Peng, S., and Sun, X., “A Straightforward Method for wall Impedance Eduction in a Flow Duct,” J. Acoust. Soc. Am., Vol. 124, No. 1, July 2008.

${ }^{15}$ Simonich, J. Narayanan, S., Morin, B., and Patrick, W., "Development and Quantification of an In-Situ Grazing Flow Impedance Measurement Technique," AIAA Paper 2005-2848, May, 2005.

${ }^{16}$ Jones, M. G., Watson, W. R., Parrott, T. L., and Smith, C. D., "Design and Evaluation of Modifications to the NASA Langley Flow Impedance Tube," AIAA Paper 2004-2837, May 2004.

${ }^{17}$ Gerhold, C., Cabell, R., and, Brown, M., "Development of an Experimental Rig for Investigation of Higher Order Modes in Ducts," AIAA Paper 2006-2637, May, 2006.

${ }^{18}$ Crocker, Malcolm J., Handbook of Noise and Vibration Control, John Wiley and Sons Inc., New York, N.Y., 2007, pp 130-134.

${ }^{19}$ Myers, M. K., "On the Acoustic Boundary Condition in the Presence of Flow," Journal of Sound and Vibration, Vol. 71, No. 3, 1980, pp. 429-434.

${ }^{20}$ Desai, C. and Abel, John F., Introduction to the finite element method: a numerical method for engineering analysis, Van Nostrand Reinhold Company, New York, N.Y., 1972.

${ }^{21}$ Intel, Intel Math Kernel Library for Linux OS Users Guide, Document NO. 314774-007US, Version-007, Aug. 2008, http://developer.intel.com
} 
${ }^{22}$ Stewart, G. W. III, “A Modification of Davidon's Minimization Method to Accept Difference Approximations of Derivatives," Journal of ACM, Vol. 14, No. 1, 1967, pp 72-83.

${ }^{23}$ Goldberg, D., Genetic Algorithms in Search, Optimization and Machine Learning, Addison-Wesley, New York, NY, 1989 , pp. $75-147$. 